\title{
BMJ Open Research priorities for young people with cancer: a UK priority setting partnership with the James Lind Alliance
}

\author{
Susie Aldiss, ${ }^{1}$ Lorna A Fern, ${ }^{2,3}$ Robert S Phillips, ${ }^{4}$ Amy Callaghan, ${ }^{5}$ Karen Dyker, ${ }^{6}$ \\ Helen Gravestock, ${ }^{7}$ Michael Groszmann, ${ }^{8}$ Leila Hamrang, ${ }^{5}$ Rachael Hough, ${ }^{9}$ \\ Demi McGeachy, ${ }^{5}$ Sue Morgan, ${ }^{10}$ Sam Smith, ${ }^{11}$ Sheela Upadhyaya, ${ }^{12}$ \\ Helen Veitch, ${ }^{11}$ Lara Veitch, ${ }^{3,5}$ Max Williamson, ${ }^{3,5}$ Jeremy S Whelan, ${ }^{\circ} 2$ \\ Faith Gibson ${ }^{1,13}$
}

To cite: Aldiss S, Fern LA, Phillips RS, et al. Research priorities for young people with cancer: a UK priority setting partnership with the James Lind Alliance. BMJ Open 2019;9:e028119. doi:10.1136/ bmjopen-2018-028119

- Pre-publication history and additional material is published online only. To view please visit the journal online (http://dx.doi. org/10.1136/bmjopen-2018028119).

Received 22 November 2018 Revised 14 May 2019 Accepted 15 May 2019

Check for updates

(c) Author(s) (or their employer(s)) 2019. Re-use permitted under CC BY-NC. No commercial re-use. See rights and permissions. Published by BMJ.

For numbered affiliations see end of article.

Correspondence to

Dr Lorna A Fern;

lorna.fern@nhs.net

\section{ABSTRACT}

Objectives To conduct a UK-wide survey of young people who have experienced cancer, carers and professionals, to identify and prioritise research questions to inform decisions of research funders and support the case for research with this unique cancer population.

Design James Lind Alliance Priority Setting Partnership. Setting UK health service and community.

Methods A steering group oversaw the initiative and partner organisations were recruited. Unanswered questions were collected in an online survey. Evidence searching verified uncertainties. An interim survey was used to rank questions prior to a final prioritisation workshop.

Participants Young people aged 13-24 years with a current or previous cancer diagnosis, their families, friends, partners and professionals who work with this population.

Results Two hundred and ninety-two respondents submitted 855 potential questions. Following a refining process and removal of 'out of scope' questions, 208 unique questions remained. Systematic evidence checking identified seven answered questions and 16 were the subject of ongoing studies. The interim survey was completed by 174 participants. The top 30 questions were prioritised at a workshop attended by 25 young people, parents and multidisciplinary professionals. The top three priorities are: (1) What psychological support package improves psychological well-being, social functioning and mental health during and after treatment? (2) What interventions, including self-care, can reduce or reverse adverse short-term and long-term effects of cancer treatment? (3) What are the best strategies to improve access to clinical trials? The remaining questions reflect the complete cancer pathway: new therapies, life after cancer, support, education/employment, relapse and endof-life care.

Conclusions We have identified shared research priorities for young people with cancer using a rigorous, personcentred approach involving stakeholders typically not involved in setting the research agenda. The breadth of priorities suggest future research should focus on holistic

\section{Strengths and limitations of this study}

- Stakeholders who do not often have a say in setting the research agenda were invited to submit research questions and prioritise them via online surveys.

- Young people, carers and clinicians agreed on the top 10 priority topics.

- We made use of the well-established and transparent James Lind Alliance methodology and provide clarity in our description of decision making in the final top 10 Prioritisation workshop.

- There was under-representation from minority ethnic groups and males.

- We are unable to determine the response rate of the survey due to the snowballing method of recruitment.

and psychosocial care delivery as well as traditional drug/ biology research.

\section{INTRODUCTION}

Young people, broadly those aged 13-24 years at diagnosis, present with a spectrum of cancers distinct from children and older adults. ${ }^{1}$ The treatment and care they receive is complex, a consequence of a disparate range of cancer types occurring during a period of unique physical and psychological growth, superimposed on the social and cultural dimensions of teenage and young adult development. This is further complicated by an extensive network of significant others, such as parents, siblings, partners and peers. In the UK, young people account for approximately 2600 new cancer cases every year, representing less than $1 \%$ of all new cancers; despite this, cancer is the leading cause of disease death in young people aged 15-24 years. ${ }^{2}$ 
There are additional factors to be considered. We already know young people's physical, psychological and social responses to a cancer diagnosis are different. For some cancers they have poorer survival than older adults and children, while other cancers demonstrate better outcomes. $^{3-5}$ Where poorer outcomes are reported, routes to diagnosis, unique host and cancer biology, responses to adult/child protocols and lesser involvement in cancer research are all factors that have been implicated ${ }^{6-10}$ We also know that caring for young people is complex; age-appropriate care has now been defined, ${ }^{11}$ and this will help services to meet the distinct needs of this population.

The research agenda for young people with cancer has typically been set by professionals and researchers (http://csg.ncri.org.uk/about-the-csgs/clinical-studiesgroups/tya-germ-cell-tumours/). In the UK, young people have prioritised research themes identified by professionals. ${ }^{12} 13$ However, working with young people and carers/significant others as equal partners to set research priorities for young people with cancer had not yet been explored. Prioritising areas for research as identified by young people and carers is crucial as increasing evidence illustrates research questions and outcomes prioritised by professionals may not be aligned to those experiencing the disease. ${ }^{14}$ Patients and carers tend to prioritise non-drug treatment research while ongoing research strategies are dominated by drug evaluations. ${ }^{15}$ This mismatch in priorities is particularly relevant for young people due to their unique psychosocial status and rarity of cancer types experienced. Furthermore, research funders increasingly ask if proposed areas of research are a priority area for patients as well as professionals.

The James Lind Alliance (JLA) bring together patients, carers and clinicians in Priority Setting Partnerships (PSPs) to identify and prioritise unanswered questions (uncertainties) in specific health conditions (http:// www.jla.nihr.ac.uk). The purpose is to inform funders of priority issues important to patients, carers and clinicians (http://www.jla.nihr.ac.uk/priority-setting-partnerships/). Initially, PSPs focused on 'treatment' related uncertainties. However, 'treatments' are now interpreted more broadly and can include causes, diagnosis, prognosis, care, support and survivorship. ${ }^{16}$

Following the JLA methodology, we aimed to conduct a UK-wide prioritisation exercise for cancer in young people. Our ultimate aim was to identify and prioritise research questions to inform decisions of research funders and support the case for research in this underserved group.

\section{METHODS}

Methodology was in concordance with the JLA process ${ }^{16}$ : the full protocol is available from: http://www.jla.nihr. ac.uk/priority-setting-partnerships/teenage-and-youngadult-cancer/. Briefly, this involved forming a steering group to define the scope and oversee progress, then seeking questions of relevance, finding which were unanswered, and prioritising these through online ranking to short-list questions to be finally prioritised in an all day, in-person workshop with key stakeholders.

\section{Ethical considerations}

Ethical approvals are not required for JLA partnerships as per JLA and National Health Services Patient Safety Agency National Research Ethics Service guidance. ${ }^{17}$

\section{Set-up}

Steering group

An expert steering group comprising all co-authors oversaw the project, approved aims/objectives, survey materials, cleaned data and provided expert opinions for evidence checking. The JLA chair (SU) provided neutral facilitation ensuring all voices were heard. The group included young people with a previous cancer diagnosis $(\mathrm{n}=5)$, healthcare and allied health professionals with a minimum of $50 \%$ patient facing role: nursing, $n=2$; youth support co-ordinator, $\mathrm{n}=1$; adult medical oncology, $\mathrm{n}=1$; adult clinical oncology, $\mathrm{n}=1$; paediatric haematology, $\mathrm{n}=1$; psychiatry, $\mathrm{n}=1$; general practitioner, $\mathrm{n}=1$, paediatric oncology, $\mathrm{n}=1$. Two funding partners were members but not involved in the final prioritisation exercise, nor were non-clinical researchers (SA, LF, FG). The steering group identified potential partners mainly charities, professional and young person networks who were approached to assist with survey dissemination ensuring wide reach of the survey.

\section{The scope}

Cancer care for young people is complex, therefore the scope was kept broad and included questions on prevention, causes, diagnosis, treatment, care, follow-up, survivorship, relapse and end-of-life (figure 1).

Our aim was, 'To identify gaps and unanswered questions in research, the answers to which may reduce the individual and societal burden of young peoples' cancer.'

\section{Process}

A summary of the complete JLA process can be seen in figure 2.

\section{Stage 1: collecting questions}

The survey was developed by the steering group and piloted with five young people, nine parents and five professionals outside the steering group. The survey was launched on 4 October 2016 and remained open until 31 December 2016. The following groups could participate:

- Anyone diagnosed with cancer between 13-24years.

- Relatives/friends/partners/carers of someone with a cancer diagnosis between 13-24years.

- Professionals working with young people aged 13-24years with cancer.

Respondents could submit up to five questions related to young people's cancer they considered unanswered. Basic demographic data were requested and a box was available for free text comments. Our partners promoted 


\section{Your questions can be about any aspect of Teenage and Young Adult Cancer during:}
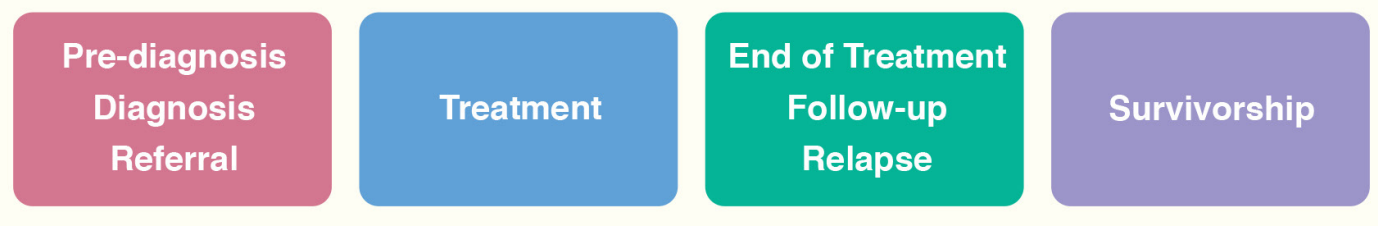

Palliative care

and end of life

Such as:

- Communication
- Relationships

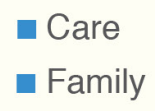

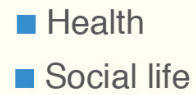

- Long-term effects
Side effects

Figure 1 Pathway of care included in the project scope.

the survey through websites, social media, newsletters and conferences including a young person's cancer conference. After 3 weeks, an interim analysis of demographic details identified under-represented groups and strategies were employed to promote the survey to those groups.

\section{Stage 2: refining questions}

Submitted questions were examined in detail and free text sections studied for further questions.

\section{Organising the questions}

Questions were coded by LF, SA and FG using the International Cancer Research Partnership Common Scientific Outline (CSO, https://www.icrpartnership.org/cso) to group questions into themes easing review and discussion. Differences in opinion in coding were resolved by the steering group. Questions which did not fit our scope, or could not be answered by research, were classed as 'out of scope' (see box 1 for categories used for out of scope questions).

Identifying out of scope questions was an iterative process, checked and agreed by the steering group. Over two meetings, steering group members were split into small groups and allocated questions within CSO themes depending on their expertise. Similar questions were merged and reworded into Population, Intervention, Comparison, Outcome (PICO) format.

\section{Evidence searching}

A data assessment group consisting of steering group members including young people and experts in evidence synthesis oversaw the evidence searches. The search strategy is available at: http://www.jla.nihr.ac.uk/ priority-setting-partnerships/teenage-and-young-adultcancer/. Searches were undertaken to identify questions answered by existing evidence. Searches were conducted during August/September 2017 and, following the JLA Guidebook, were restricted to the last 5 years (2012). Publications were relevant if they brought evidence from multiple studies together, for example, systematic reviews and qualitative meta-synthesis or were based on large, nationally representative cohort studies. As evidence was unlikely to be specific to our cohort of 13-24years, evidence including older and younger populations was considered and the data assessment group/steering group debated whether further work focussing specifically on the age of our cohort was required. Searches were also conducted for ongoing studies through clinical trial databases and personal communication with experts.

\section{Stage 3: question prioritisation Interim survey preparation}

Following the steps of the JLA Guidebook, there were too many unanswered questions to be included in the interim survey. The steering group considered all questions asked by more than one person to be high-ranking and were included in the interim survey, the remaining questions were then rated anonymously by the steering group with the top 104 scoring questions included in the interim survey.

\section{Interim survey}

The wording of these questions was checked by young people on the steering group. The survey opened in November 2017 for 3 weeks, publicised as in the initial survey. In addition, initial survey participants who left contact details for future participation were emailed directly.

Respondents were invited to rate the 104 questions. The options for rating were: very low priority (score 1), low priority (score 2 ), high priority (score 3 ), very high priority (score 4), no opinion (no score). Respondents were asked to select 'no opinion' if they were unsure about a question's priority or had no opinion. Questions were grouped into sections:

1. Causes of cancer, prevention and diagnosis.

2. Treatments/therapies.

3. Short/long-term side effects.

4. Information/support.

5. Psychological support.

6. How cancer impacts on daily life.

7. Impact of cancer on families, friends and partners.

8. End of treatment and follow-up.

9. Healthcare delivery.

10. End-of-life care. 


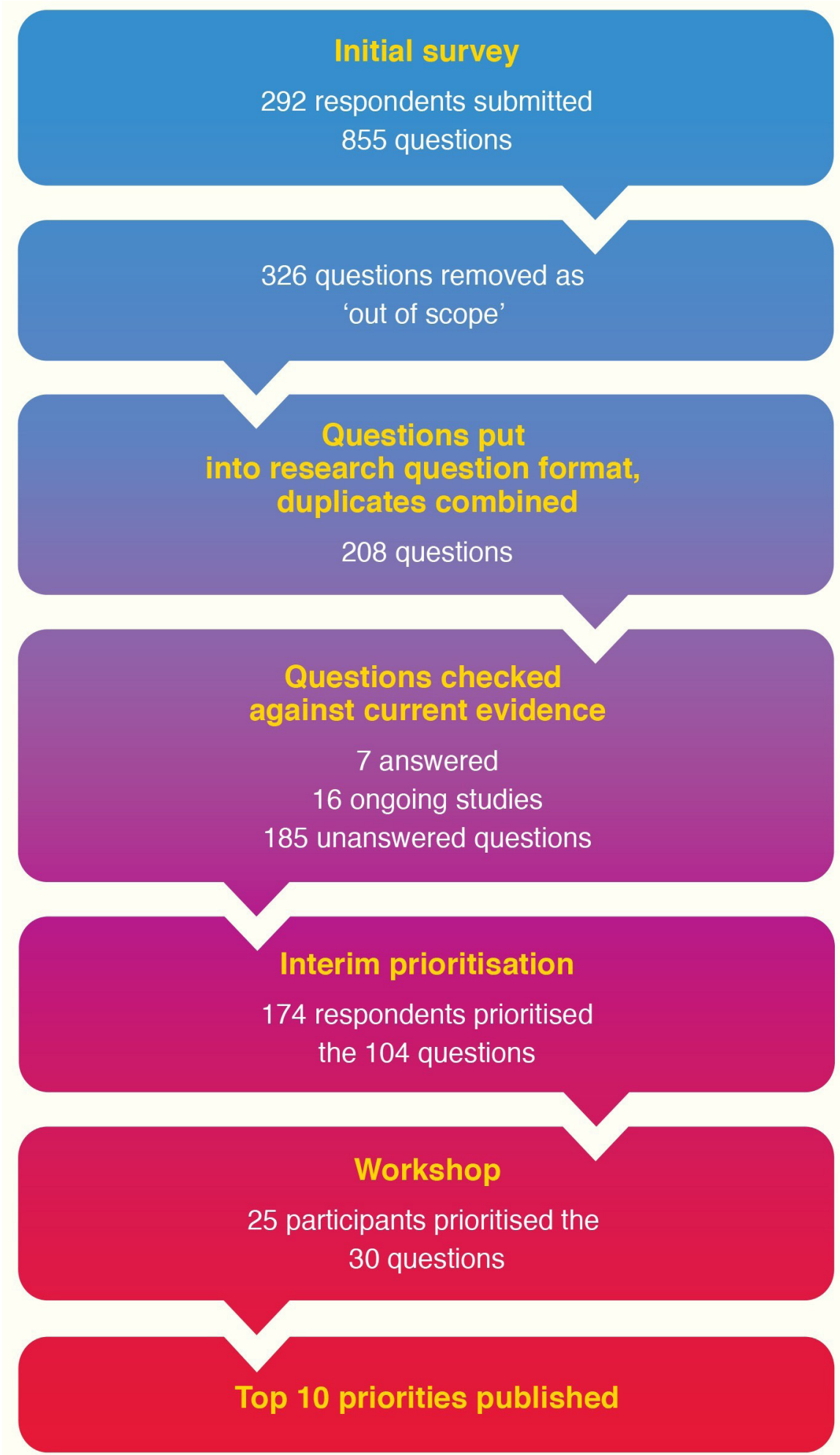

Figure 2 Overview of the Teenage and Young Adult Cancer Priority Setting Partnership methodology and results.

Survey fatigue was minimised by randomisation of section order.

To account for missing data, average scores for each question were calculated for each group: patients/ former patients, family members/friends/partners and professionals. The questions were then ordered from highest to lowest score for each group. The steering group reviewed ratings between groups. As the distribution of respondents from the three groups was not equal, a simple ranking from combined scores would excessively weight professionals' views. The average ranks within each groups was calculated, then combined to give the final rank. The top 30 questions were short-listed for further prioritisation. 


\section{Box 1 Out of scope question categories and examples}

1. The question did not fit the scope of reducing the individual and societal burden of young peoples' cancer or could not be answered by research.

- 'Can a cancer sufferer become an organ donor?'

2. It was a statement rather than question (and no specific question could be identified from the statement).

- 'Can the late effects Drs stop telling us how BAD outcomes are and focus just a little on some of the POSITIVE outcomes'.

3. The question was ambiguous, was interpreted in different ways by steering group members and the meaning could not be resolved following discussion.

- 'Supportive care'.

4. The focus was on research methods rather than a research topic.

- 'How should we collect information about the late-onset side effects of cancer treatment in Teenagers and Young Adults?'

5. The question related to a specific person's situation/issue.

- 'Who can I talk to about my worries for my child?'

6. It was a political statement.

- 'Should medical professionals routinely explain to patients that there may be more up-to-date treatments available in other parts of the world, which may increase the patients' chances of survival?'

\section{Stage 4: top 10 prioritisation}

The top 10 prioritisation workshop took place in January 2018. Young people were invited from the steering group, self-selected survey respondents and the BRIGHTLIGHT Young Advisory Panel (www.BRIGHTLIGHTSTUDY. COM). Parents were recruited through CLIC Sargent (a UK charity) and self-selected survey participants. Professionals on the steering group with a patient facing role could participate; other professionals were identified by the steering group.

Prior to the workshop, participants were asked to individually rank the 30 questions in order of their importance. The workshop was chaired by SU and supported by two JLA facilitators. Participants were split into three pre-allocated groups ensuring a balance of multidisciplinary professionals, young people and parents. In each group, participants shared their three highest and lowest ranking questions. Discussion followed, and the groups ordered the 30 questions from highest to lowest. During the first break, ranking of the 30 questions from the three groups were combined. In the following session, in new group compositions, the combined consensus ranking was the discussion starting point. Following this second round of discussion, the group rankings were again collated and all participants formed one group to debate and agree the top 10. Two silent observers attended each of the three workshops; each pair included one member of the co-ordinating team (FG, SA and LF). The six observers took notes of decision making processes during the workshop but did not interact with the participants in any way.

\section{Patient and public involvement}

Young people were involved as equal members of the steering group, directing the study, developing and agreeing the scope, reviewing and agreeing questions, and were involved in all stages of the prioritisation process. The surveys invited responses from patients and carers. Young people and parents attended the final prioritisation workshop alongside professionals as equal stakeholders.

\section{RESULTS}

\section{Initial survey}

Two hundred and ninety-two people submitted 855 questions. Respondents included 108 (36\%) young people, $101(34 \%)$ family members/partners/friends and 83 professionals $(30 \%)$. The majority of family members/ partners/friends described themselves as parent/carer, $(\mathrm{n}=71 ; 70 \%), 13(13 \%)$ as relative, $13(13 \%)$ friends and four partners $(4 \%)$. A range of multidisciplinary professionals responded; equal numbers of nurses and doctors $(\mathrm{n}=25,30 \%), 24(29 \%)$ allied health professionals and nine $(11 \%)$ 'other'. 'Other' included third sector (charity and not for profit organisations) professionals and academic researchers. The cancer types experienced by young people responding was broadly similar to incident cases. Supplementary material one shows respondent demographics.

Three hundred and twenty-six out of scope questions were removed, box 1 illustrates examples. Following combining similar questions and applying PICO formatting, 208 questions remained.

\section{Analysis of uncertainties}

Seven questions were considered as already answered, and 16 questions were the focus of ongoing studies. For the majority of questions, $(n=185)$, no reviews or ongoing studies were identified. Where identified reviews only partly answered the question, these were recorded as unanswered. The steering group discussed all questions ensuring a consensus agreement of answered/unanswered questions.

\section{Interim survey}

One hundred and eighty-five questions were unanswered. Sixty-four of these were submitted by more than one person and therefore included in the interim survey along with the top 40 questions rated by the steering group, resulting in 104 questions in the interim survey.

Ratings were submitted by 174 respondents: 58 $(33 \%)$ young people, 45 (26\%) family members/partners/friends and 71 professionals $(41 \%)$. In the family members/partners/friends group, most were parents/ carer, $(\mathrm{n}=34,76 \%)$, with eight $(18 \%)$ relatives, three friends and partners $(6 \%)$. See supplementary material two for respondent demographics. More nurses $(n=27$, $38 \%)$ responded than medical doctors $(\mathrm{n}=17,24 \%)$. Fourteen (20\%) respondents were allied health professionals and $11(15 \%)$ selected 'other' (missing data, $\mathrm{n}=2$, $3 \%)$. 


\section{Workshop}

Twenty-five participants attended: seven young people, four parents and 14 multidisciplinary professionals including nursing, oncology, haematology, social work, youth-support, psychiatry and physiotherapy. Seven participants were steering group members. Three young people and one parent sent late apologies. Supplementary material three shows schematic diagram of workshop.

\section{Top 10 prioritisation strategies}

Despite working independently, the three groups applied similar prioritisation strategies:

\section{(1) Including all parts of the care pathway}

Groups wanted the top 10 priorities to encompass the cancer pathway including diagnosis, new treatments, end-of-treatment, support, relapse, life beyond cancer and end-of-life care. They also wanted to reflect the range of professionals and diverse experiences of young people and parents attending.

\section{(2) Ensuring all themes were represented}

Groups clustered questions into similar themes, such as support, treatment/side effects, with an aim to include 'themes' across the pathway. This meant choosing one question from each theme, as multiple questions from one theme would sacrifice prioritisation of a question in a different area. As an example, choosing 'What General Practitioner (GP) or young person strategies, such as awareness campaigns and education, improve early diagnosis for young people with suspected cancer?' or, 'What are the most effective strategies for engaging primary care professionals (eg, GPs) to listen to young people?'

\section{(3) Choosing questions that could include other questions/overlap}

Linked to the earlier point, the groups selected overlapping questions which could be answered within other questions. For example, 'What are the best ways of supporting a young person who has incurable cancer?' could include questions, 'For young people with incurable cancer, how should parents/carers communicate with them to improve quality of life and experience?' and 'For young people with incurable cancer, how should healthcare professionals communicate with them to improve quality of life and patient experience?' as communication could be considered within 'support'.

\section{(4) Considering impact and generalisability of questions}

Prior to the workshop, participants prioritised their top 3 questions based on personal experience or work area. However, during the workshop, participants were observed to reflect on their opinions and a consensus emerged that the top 10 should be wide reaching and avoid focusing on single cancers or treatments. For example, there was consensus on prioritisation of side-effects and 'What interventions, including self-care, can reduce or reverse adverse short-term and long-term effects of cancer treatment?' was selected over questions which focused on one side-effect or treatment such as 'What are the long-term physical effects of stem cell transplants, how long do they last and how could they be reduced?'.

\section{(5) Questions which focus on support for families}

Including a question on family support was considered a priority and it was agreed the question, 'What psychological support package improves psychological well-being, social functioning and mental health during and after treatment?' should include supporting families.

\section{(6) Prioritising interventions over descriptive questions}

The groups were united in their view of the utility of descriptive studies and prioritised interventions, for example, 'What psychological support package improves psychological well-being, social functioning and mental health during and after treatment?' was prioritised over 'How common is psychological distress and/or mental health problems in young people following treatment?', stating that the latter question would naturally be addressed by the processes involved in developing the intervention.

\section{(7) Excluding questions considered to be too subjective to be answered by research}

Some questions were considered too individual and/or subjective to address. For example, 'What are the factors that should determine stopping treatment when the young person cannot be cured?', participants agreed this was person-dependant and too variable to generate a 'tick list' of factors which would determine stopping treatment. Similarly, within the same theme, 'For young people with incurable cancer, how should healthcare professionals communicate with them to improve quality of life and patient experience?' was considered to be subjective, with participants agreeing its importance but also a difficult skill to teach to professionals and was manifestation of a wider social problem of our views on death and dying.

The final group exercise generated considered debate around the order of questions, and terminology, however the top 3, remained consistent after the first part of the workshop. Of note, a number of questions underwent slight wording changes; at the request of young people 'self-care' was included as an intervention for priority number 2 and the word 'normal' was removed from one of the questions in the remaining top 20. Healthcare professionals requested, 'specialist care' be replaced with 'Principal Treatment Centre' in question 6. The final agreed, top 10 priorities for young people with cancer are shown in box 2 and box 3 lists the remaining 20 questions discussed at the workshop but not placed in order of priority.

\section{DISCUSSION}

The Teenage and Young Adult Cancer PSP has brought together for the first time, young people, carers and professionals to prioritise research questions focused on young people with cancer. The top 10 priorities provide 
Box 2 Top 10 research priorities for teenage and young adult cancer

1. What psychological support package improves psychological well-being, social functioning and mental health during and after treatment?

2. What interventions, including self-care, can reduce or reverse adverse short-term and long-term effects of cancer treatment?

3. What are the best strategies to improve access to clinical trials?

4. What General Practitioner or young person strategies, such as awareness campaigns and education, improve early diagnosis for young people with suspected cancer?

5. What are the best ways of supporting a young person who has incurable cancer?

6. What are the most effective strategies to ensure that young people who are treated outside of a young person's principal treatment centre receive appropriate practical and emotional support?

7. What interventions are most effective in supporting young people when returning to education or work?

8. How can parents/carers/siblings/partners be best supported following the death of a young person with cancer?

9. What is the best method of follow-up and timing which causes the least psychological and physical harm, while ensuring relapse/ complications are detected early?

10. What targeted treatments are effective and have fewer short-term and long-term side-effects?

a much-needed resource to inform research funding decisions in government and charitable agencies. The priorities highlight the need for research strategies to be holistic in their approach rather than driven by biological and drug intervention research. The top research priority was identified as, 'What psychological support package improves psychological well-being, social functioning and mental health during and after treatment?' The top 10 priorities include the complete cancer 'timeline': finding biological targets; early diagnosis; access to new treatments; supporting families; returning to education/ employment; minimising the long-term effects of treatment; supporting young people whose cancer cannot be cured; and supporting bereaved families and carers. While the majority of questions are related to the healthcare setting, the breadth of the questions and those in the remaining 20 include communication; relationships; service provision; social and educational milestones, thus suggesting that research funding could also be sought from social and educational funding bodies. It is now critical that funders and researchers ensure that future research focuses on what is important to young people, carers and professionals.

In a previous consultation exercise in which young people rated research topics already identified by professionals, improving time to diagnosis was prioritised as the most important area of research, followed by late-effects and survivorship. ${ }^{12}$ Quality-of-life was rated as important as survival by $75 \%$ of the young people participating and they also wanted research to include the effect of cancer on family and friends. ${ }^{12}$ These priorities are all reflected in the top 10 .
Box 3 The additional 20 questions taken to the workshop (not listed in order of priority)

\section{Diagnosis}

- What factors affect the time to diagnosis and what outcomes are affected?

- What are the most effective strategies for engaging primary care professionals (eg, general practitioners) to listen to young people?

\section{Treatment}

- What key factors (both cancer and individual) determine whether a treatment plan for children or adults will give better outcomes?

- What is the best treatment for brain cancers to increase survival and decrease toxicity?

-What are the factors that predict life threatening chemotherapy side effects?

\section{After treatment}

-What can young people do to help their recovery after chemotherapy or radiotherapy?

- What are the long term physical effects of a cancer diagnosis and treatment and how long do they last?

- What are the long-term physical effects of stem cell transplants, how long do they last and how could they be reduced?

- How common is psychological distress and/or mental health problems in young people following treatment?

- What causes problems with cognitive functioning ('chemobrain'), how long do they last and what are the most effective treatments and strategies?

- At the end of treatment and during long term follow up, what support services improve psychological well-being, social functioning and mental health?•

-What are the best ways to support young people getting back to 'everyday' life after treatment?

- What interventions are most effective in supporting young people who are experiencing fatigue/tiredness when returning to work or education?

- What are the best strategies for detecting and treating second primary cancers early?

\section{Relapse}

- What is the most effective way of supporting young people with relapsed cancer?

\section{End of life}

- What are the factors that should determine stopping treatment when the young person cannot be cured?

- For young people with incurable cancer, what methods, techniques or strategies for communication can help them to talk with their family and friends about their situation?

- For young people with incurable cancer, how should healthcare professionals communicate with them to improve quality of life and patient experience?

- For young people with incurable cancer, how should parents/carers communicate with them to improve quality of life and experience?

- What are the support needs of the family following the death of a young person with cancer? The remaining 20 priorities discussed at the workshop

The top priority identified in this exercise reflects an increasing drive to focus on both physical and psychological health during and after cancer. ${ }^{18}$ It is increasingly recognised that a cancer diagnosis has serious implications 
for young people's mental health but this has yet to be systematically investigated; a recent UK survey reported $79 \%$ of young people felt cancer seriously impacted their emotional well-being, $70 \%$ of young people reported depression during treatment and 90\% experienced anxiety during treatment. ${ }^{19}$ How these young people are best supported is currently unknown.

A number of cancer-related PSPs exist, however, this is the first PSP to focus on an 'age' rather than a 'cancer type' and the whole cancer pathway. The recent Living with and Beyond Cancer PSP reflects similar priorities as the Teenage and Young Adult Cancer PSP highlighting the need to have a holistic approach to research undertaken, that focusses on psychological impact, understanding and managing side-effects, and information that helps those with cancer to make life-style choices that help them to live their lives fully (https://www.ncri.org. $\mathrm{uk} / \mathrm{lwbc} /$ ).

\section{Challenges, strengths and limitations}

We encountered several challenges. Our time to complete was 3 years versus an anticipated 12-18 months. The appointment of a dedicated researcher who also administered steering group meetings, agendas, minutes and travel arrangements was critical to the project's final completion. The project was resource intensive, requiring input from all steering group members. This was challenging when involving clinicians with full schedules, and young people in full-time employment/education. Further to this, the scope of the PSP was kept broad to reflect the diverse care pathways and needs of young people with cancer and we also considered questions relating to families and extended networks whereas other PSPs have excluded questions which are not patient-focused. ${ }^{20}$ The wide scope generated increased work for sorting and handling more diverse questions which often did not neatly fit the 'PICO' format recommended by the JLA.

The use of the rigorous and transparent JLA method enhances the validity of the process and results. It is notable that the proportion of respondents from each of the three groups (young people, carers and professionals) submitting questions was about the same, and similar themes from their questions was reflected across these groups. Young people were involved in shaping the project from the outset, as members of the steering group. Their input was key; for example, they helped to ensure the surveys were presented in a user-friendly format and appropriate routes to dissemination were used. Young people all reported a positive experience of being involved in the steering group,

If you have been affected by cancer, whether that's as a young person or have been involved in their care as a parent, carer or professional, you will know that there are a lot of unanswered questions. As a survivor, my main priority is around long-term effects from cancer treatment and I'm glad that this question made it in the Top 10. It has been a privilege to be involved in this project, I'm looking forward to seeing change within the young people's services whether that's at the point of diagnosis, during treatment or survivorship." Comment from young person on steering group

Further to this, we know that the acceptability of research questions influences professionals' decisions to offer study entry and young person's likelihood to enrol in a study ${ }^{8}$; we have a collective list of research questions 'acceptable' to both professionals and young people.

Despite the projects' strengths, we acknowledge limitations. We do not know the response rate of survey uptake given the snowballing method of recruitment. The majority of young people and carers were white and female. This was in spite of targeting charities and user groups which focus on males and minority groups. This is typical with survey research and many other PSPs have reported a low proportion of responses from males and people from a range of ethnicities. ${ }^{21}{ }^{22}$ Further research could explore interventions to encourage the participation of these groups in such surveys to maximise the chance of the voices of these under-represented cohorts being heard. We also did not explore gender orientation beyond 'male/female/other/prefer not to answer' during data collection or in the workshop. On searching the internet young people on the PSP identified 75 terms for gender, it was their suggestion that to reduce discrimination we should leave an option 'other' with free text option. '. However questions related to lesbian, gay, bisexual and transgender young people were submitted and can be viewed in the full report. We would encourage researchers to include the needs of these groups during protocol development and in designing recruitment strategies. Very few young people currently on treatment took part; however, we know from other research that during the treatment phase young people will approach their treatment team for answers to questions and it is when nearing the end of treatment that young people start to consider additional questions. ${ }^{23}$ Similar to other PSPs involving children and young people, ${ }^{20}$ the younger end of our cohort were less well represented (aged 13-15years) and future PSPs involving young people may need to consider alternative data collection methods for this group.

\section{IMPLICATIONS}

The uncertainties raised and prioritised are the outcome of a systematic and transparent process with stakeholders who have not often had a voice in setting the research agenda. When selecting the questions to be included in the top 10, the workshop participants intentionally opted for broad questions, in order to capture the wide range of issues. For some questions, further refinement is required to take the topic forward as a feasible research project. Many questions encompass a range of interventions requiring a programmatic approach to research reflecting 
growth of the community and are ideally placed for major research funding calls. ${ }^{24}$ For these, specific interventions, comparators and outcomes require identification along with decisions made about how and when outcomes are measured.

Many questions submitted in the survey were considered to be outside the scope of this initiative. Examples include queries about availability of services or information, questions about research methodology and personal questions relating to someone's particular situation. The steering group considered these questions to be important and were determined to ensure these submissions were not 'lost'. Further analysis of these out of scope questions is under way as they highlight gaps in information provision indicating that better signposting for young people and families is required.

\section{CONCLUSION}

For too long now, the research funding landscape has overlooked young people with cancer, prioritising research in the main cancer types occurring in adults. Our community is further disadvantaged by the absence of young person specific research funding bodies. Despite these real challenges, we now have available for funders, researchers, clinicians and the wider community, systematic evidence of the priority areas of research, confirmed as unanswered and defined as important by young people, their networks and the healthcare professionals caring for them. In the UK, other PSPs have generated themed calls from major funders. ${ }^{25}$ As a community, we eagerly await the response of these same institutions to the identified research priorities in this typically underserved population.

\section{Author affiliations}

${ }^{1}$ School of Health Sciences, University of Surrey, Guildford, UK

${ }^{2}$ Oncology Division, University College London Hospitals NHS Foundation Trust, London, UK

${ }^{3}$ Teenage, Young Adult and Germ Cell Clinical Studies Group, National Cancer Research Institute, London, UK

${ }^{4}$ Centre for Reviews and Dissemination, University of York, York, U.K.

${ }^{5}$ Teenage and Young Adult Cancer Priority Setting Partnership steering group, London, UK

${ }^{6}$ Oncology, Level 4, Bexley Wing, St James' University Hospital, Leeds, UK ${ }^{7}$ Policy and Campaigning, CLIC Sargent, London, UK

${ }^{8}$ Paediatric \& Adolescent Division, University College London Hospitals NHS Foundation Trust, London, UK

${ }^{9}$ Children and Young Peoples Cancer Service, University College London Hospitals NHS Foundation Trust, London, UK

${ }^{10}$ Teenage and Young Adult Cancer Service, Leeds General Infirmary, Leeds, UK

${ }^{11}$ Service Delivery, Teenage Cancer Trust, London, UK

${ }^{12}$ The James Lind Alliance, National Institute for Health Research Evaluation, Southampton, UK

${ }^{13}$ Centre for Outcomes and Experience Research in Children's Health, IIIness and Disability (ORCHID), Great Ormond Street Hospital For Children NHS Foundation Trust, London, UK

Acknowledgements The Teenage and Young Adult Cancer PSP would like to thank all the young people, their carers, families, friends and partners who took the time to send in their questions and rate the importance of them. Thank you also to the young people, parents and professionals who attended the final workshop.
We would also like to extend our thanks to our partners who distributed the survey through their websites, newsletters and social media channels.

Contributors All authors were part of the Teenage and Young Adult Cancer Priority Setting Partnership steering group or coordinating team and made substantive contributions to the conduct of the study, overseeing all aspects of the work. All authors, SA, LF, RP, AC, KD, HG, MG, LH, RH, DM, SM, SS, SU, HV, LV, MW, JSW and $F G$ contributed to protocol design, survey refining, data cleaning and refining questions submitted in the initial survey. The project was managed by LF, SA, FG and SU. Specific contributions included: survey design (SA), coding the survey submissions (FG, LF, SA), searching and checking uncertainties (SA, LF, RP), managing data entry (SA, LF). All authors reviewed and approved the final version of this paper.

Funding This work was supported by: Teenage Cancer Trust, CLIC Sargent and Children with Cancer UK. LF is funded by Teenage Cancer Trust.

Competing interests None declared.

Patient consent for publication Not required.

Provenance and peer review Not commissioned; externally peer reviewed.

Data sharing statement Further data regarding the source of original submissions to the survey are available from: http://www.jla.nihr.ac.uk/priority-settingpartnerships/teenage-and-young-adult-cancer/

Open access This is an open access article distributed in accordance with the Creative Commons Attribution Non Commercial (CC BY-NC 4.0) license, which permits others to distribute, remix, adapt, build upon this work non-commercially, and license their derivative works on different terms, provided the original work is properly cited, appropriate credit is given, any changes made indicated, and the use is non-commercial. See: http://creativecommons.org/licenses/by-nc/4.0/.

\section{REFERENCES}

1. Birch JM, Alston RD, Kelsey AM, et al. Classification and incidence of cancers in adolescents and young adults in England 1979-1997. Br J Cancer 2002;87:1267-74.

2. CR UK. Young People's Cancers Statistics. 2018 https://www. cancerresearchuk.org/health-professional/cancer-statistics/statisticsby-cancer-type/young-peoples-cancers (accessed 27 Sep 2018).

3. Bleyer A, Tai E, Siegel S. Role of clinical trials in survival progress of American adolescents and young adults with cancer-and lack thereof. Pediatr Blood Cancer 2018;65:e27074.

4. Smith MA, Seibel NL, Altekruse SF, et al. Outcomes for children and adolescents with cancer: challenges for the twenty-first century. $J$ Clin Oncol 2010;28:2625-34.

5. Trama A, Bernasconi A, McCabe MG, et al. Is the cancer survival improvement in European and American adolescent and young adults still lagging behind that in children? Pediatr Blood Cancer 2019;66:e27407.

6. Bleyer A, Barr R, Hayes-Lattin B, et al. The distinctive biology of cancer in adolescents and young adults. Nat Rev Cancer 2008;8:288-98.

7. Boissel N, Auclerc MF, Lhéritier V, et al. Should adolescents with acute lymphoblastic leukemia be treated as old children or young adults? Comparison of the French FRALLE-93 and LALA-94 trials. J Clin Oncol 2003;21:774-80.

8. Fern LA, Lewandowski JA, Coxon KM, et al. Available, accessible, aware, appropriate, and acceptable: a strategy to improve participation of teenagers and young adults in cancer trials. Lancet Oncol 2014;15:e341-e350.

9. Herbert A, Lyratzopoulos G, Whelan J, et al. Diagnostic timeliness in adolescents and young adults with cancer: a cross-sectional analysis of the BRIGHTLIGHT cohort. Lancet Child Adolesc Health 2018;2:180-90.

10. Lethaby CD, Picton S, Kinsey SE, et al. A systematic review of time to diagnosis in children and young adults with cancer. Arch Dis Child 2013;98:349-55.

11. Lea S, Taylor RM, Martins A, et al. Conceptualizing age-appropriate care for teenagers and young adults with cancer: a qualitative mixedmethods study. Adolesc Health Med Ther 2018;9:149-66.

12. Fern LA, Ashton J, Brooman K, et al. Which research priorities are defined by young people with cancer- second consultation by the National Cancer Research Institutes Teenage and Young Adult Core Consumer Group. Poster presented at the 2009 NCRI Conference 2010 http://abstracts.ncri.org.uk/abstract/whichresearch-priorities-are-defined-by-young-people-with-cancer- 
second-consultation-by-the-national-cancer-research-institute $\%$ C2\%92s-teenage-and-young-adult-core-consumer-group-4/ (accessed 4 Oct 2018).

13. Fern LA, Ashton J, Brooman $\mathrm{K}$, et al. Involving young people with cancer in research The National Cancer Research Institutes Teenage and Young Adult (TYA) Core Consumer Group (CCG) - a new and evolving model. Poster presented at the 2009 NCRI Conference 2009 http://abstracts.ncri.org.uk/abstract/involving-young-peoplewith-cancer-in-research-the-national-cancer-research-institutesteenage-and-young-adult-tya-core-consumer-group-ccg-a-newand-evolving-model/ (accessed 4 Oct 2018).

14. Chalmers I, Atkinson P, Fenton $\mathrm{M}$, et al. Tackling treatment uncertainties together: the evolution of the James Lind Initiative, 2003-2013. J R Soc Med 2013;106:482-91.

15. Crowe S, Fenton M, Hall M, et al. Patients', clinicians' and the research communities' priorities for treatment research: there is an important mismatch. Res Involv Engagem 2015;1:2.

16. Alliance JL. The James Lind Alliance Guidebook. Version 7. 2018 http://www.jla.nihr.ac.uk/jla-guidebook/downloads/Print-JLAguidebook-version-7-March-2018.pdf (accessed 27 Sep 2018).

17. Involve THRA. Public involvement in research and research ethics committee review. 2016 http://www.invo.org.uk/posttypepublication/ public-involvement-in-research-and-research-ethics-committeereview/ (accessed 29 Oct 2018).
18. All Party Parliamentary Group on Children TaYAwC. Listen Up! What matters to young cancer patients. $2018 \mathrm{https} / / \mathrm{www}$. teenagecancertrust.org/listenup (accessed 4 Oct 2018).

19. Sargent C. Hidden Costs. The mental health impact of a cancer diagnosis on young people. $2017 \mathrm{https} / /$ www.clicsargent.org.uk/ content/hidden-costs (accessed 4 Oct 2018).

20. Morris C, Simkiss D, Busk M, et al. Setting research priorities to improve the health of children and young people with neurodisability: a British Academy of Childhood Disability-James Lind Alliance Research Priority Setting Partnership. BMJ Open 2015;5:e006233.

21. Gadsby R, Snow R, Daly AC, et al. Setting research priorities for Type 1 diabetes. Diabet Med 2012;29:1321-6.

22. Layton A, Eady EA, Peat M, et al. Identifying acne treatment uncertainties via a James Lind Alliance Priority Setting Partnership. BMJ Open 2015;5:e008085.

23. Lea S, Martins A, Morgan S, et al. Online information and support needs of young people with cancer: a participatory action research study. Adolesc Health Med Ther 2018;9:121-35.

24. Stark D, Fern LA, Gibson F, et al. Transitioning adolescent and young adult cancer care research out of its adolescence. Eur J Cancer Care 2018;27:e12962.

25. Gibson F. How Far Do Research Priority Setting Exercises Influence What Research Is Undertaken: A Little, a Lot, or Not at All? Cancer Nurs 2019;42:89-90. 